\title{
Polarized dust emission of magnetized molecular cloud cores
}

\author{
J. Gonçalves ${ }^{1,2}$, D. Galli ${ }^{2}$, and M. Walmsley ${ }^{2}$ \\ ${ }^{1}$ Centro de Astronomia e Astrofísica da Universidade de Lisboa, Tapada da Ajuda, 1349-018 Lisboa, Portugal \\ 2 INAF - Osservatorio Astrofisico di Arcetri, Largo E. Fermi 5, 50125 Firenze, Italy \\ e-mail: goncalve@arcetri.astro.it
}

Received 13 July 2004 / Accepted 7 October 2004

\begin{abstract}
We compute polarization maps for molecular cloud cores modeled as magnetized singular isothermal toroids, under the assumption that the emitting dust grains are aspherical and aligned with the large-scale magnetic field. We show that, depending on the inclination of the toroid with the line-of-sight, the bending of the magnetic field lines resulting from the need to counteract the inward pull of gravity naturally produces a depolarization effect toward the centre of the map. We compute the decrease of polarization degree with increasing intensity for different viewing angles and frequencies, and we show that an outward increasing temperature gradient, as expected in starless cores heated by the external radiation field, enhances the decrease of polarization. We compare our results with recent observations, and we conclude that this geometrical effect, together with other mechanisms of depolarization, may significantly contribute to the decrease of polarization degrees with intensity observed in the majority of molecular cloud cores. Finally, we consider the dependence of the polarization degree on the dust temperature gradient predicted for externally heated clouds, and we briefly comment on the limits of the Chandrasekhar-Fermi formula to estimate the magnetic field strength in molecular cloud cores.
\end{abstract}

Key words. ISM: clouds - ISM: dust, extinction - magnetic fields - polarization

\section{Introduction}

Mapping the polarization of the thermal emission of dust at millimitre or submillimetre wavelengths (usually $\lambda=850 \mu \mathrm{m}$ or $1.3 \mathrm{~mm}$ ) is the principal means of probing the magnetic field geometry in molecular cloud cores. A frequent characteristic of these observations is the decrease of polarization degree $p$ as a function of the total observed intensity $I$. Usually $p$ decreases with increasing intensity $I$ with a power-law behavior, from a maximum value of $\sim 15 \%$ to about the observable limit of $\sim 1 \%$. This depolarization effect (sometimes referred to as "polarization hole", or "polarization limb brightening") has been observed in many dense cores and filamentary clouds. Recent examples include the OMC-3 region of the Orion A filamentary molecular cloud where $p \propto I^{-0.7}$ (Matthews \& Wilson 2000), several dense cores in the dark cloud Barnard 1, where $p \propto I^{-0.8}$ (Matthews \& Wilson 2002), Bok globules mapped by Henning et al. (2001) where $p \propto I^{-0.6}$, and dense cores mapped interferometrically by Lai et al. (2002), where $p \propto I^{-0.8}$. In some cases, the decrease of polarization with intensity is quite steep: for example, Crutcher et al. (2004) find $p \propto I^{-1.2}$ in the dark cloud L183, implying that not only the polarization degree but also the polarized intensity $I_{p}=p I$ decreases toward the centre of this cloud. A similar situation is apparently found also in the starless core L1544 (Ward-Thompson et al. 2000).

The dark cloud Barnard 1 is an excellent example of the type of object of interest to us. This cloud, mapped with
SCUBA by Matthews \& Wilson (2002), has dimensions of roughly $0.2 \times 0.4 \mathrm{pc}$, densities of a few times $10^{4} \mathrm{~cm}^{-3}$, and temperatures of order $10 \mathrm{~K}$. These appear unfavorable characteristics for aligning grains (see e.g. Lazarian et al. 1997). Nevertheless, B1 exhibits extremely uniform polarization at a level of around 5\% and with an extremely uniform position angle. This last point in our opinion is a strong argument in favor of an essentially uniform and likely dominant magnetic field over most of the core. However, there are exceptions to the rule in the shape of four high density (of order $10^{6} \mathrm{~cm}^{-3}$ ) prestellar or protostellar "inclusions" of dimensions $\sim 0.03 \mathrm{pc}$ (B1-a,b,c,d) apparently embedded within the region of the "general core" referred to above. These extremely high density cores also surprisingly show evidence for polarization, albeit at a lower level and with different position angles than the general core. In particular, in the core B1-c the polarization degree is very low and almost uniform over the whole observed intensity range (Matthews \& Wilson 2002) ${ }^{1}$. For illustration purposes, the $p-I$ relations measured in three well studied cores (L183,

\footnotetext{
${ }^{1}$ We note however parenthetically that the observations of polarization at high densities $\left(n \sim 10^{6} \mathrm{~cm}^{-3}\right)$ where radiation fields can hardly be of importance represent a serious challenge to grain alignment theorists. In fact, recent work shows that radiative torques due to starlight are required to drive grains to the suprathermal rotation rates necessary to minimize the disaligning effect of random collisions (Draine \& Weingartner 1996, 1997).
} 


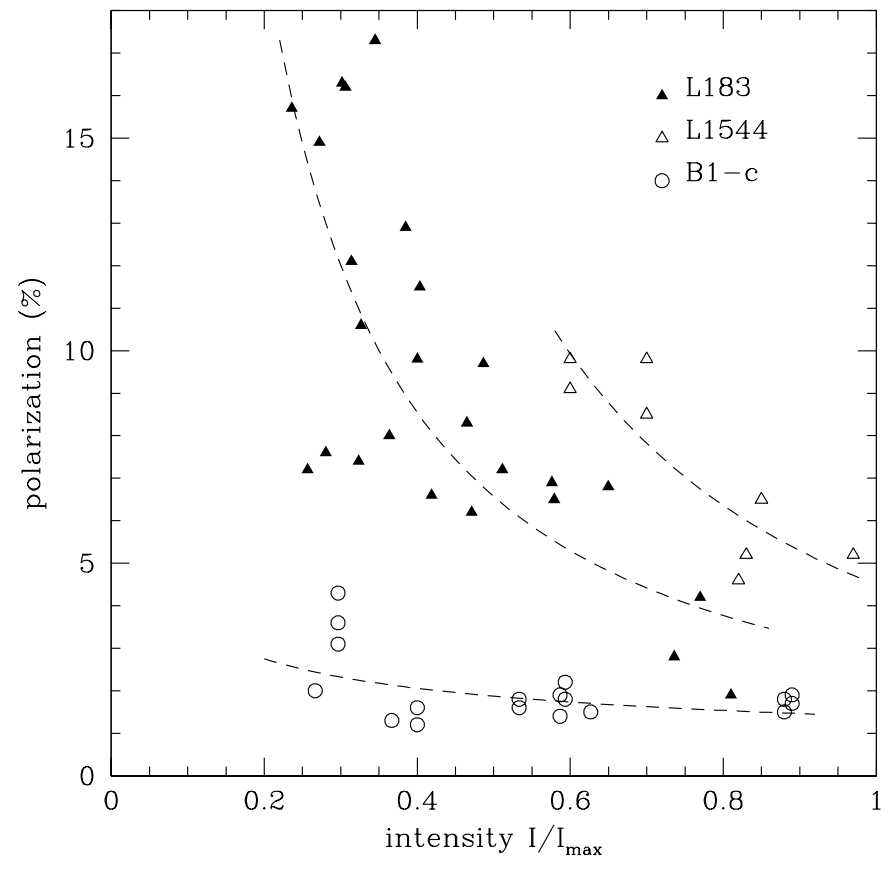

Fig. 1. Distribution of percentage of polarization $p$ vs. intensity $I / I_{\max }$ at $\lambda=850 \mu \mathrm{m}$, normalized to its maximum value, for the starless cores L183 (filled triangles, from Crutcher et al. 2004), L1544 (empty triangles, from Ward-Thompson et al. 2000), and the B1-c core (circles, from Matthews \& Wilson 2002). The dashed lines are power-law fits, with slopes -1.6 for L1544, -1.2 for L183, and -0.4 for B1-c.

L1544, and B1-c) are collected in Fig. 1, together with powerlaw fits of the original data.

The observed decrease of polarization with increasing intensity has been attributed to changes in the dust grain optical properties or shapes in the cold cores interiors due to graingrowth (Vrba et al. 1993; or Hildebrand et al. 1999): not only are bigger grains more difficult to align than smaller grains, but also the agglomeration process may make grains more spherical, thus further reducing the alignment efficiency.

All these effects are difficult to quantify. A larger density tends in general to dealign grains (Lazarian et al. 1997), but the enhanced magnetic field strength should have the opposite effect. On the basis of the Davis \& Greenstein (1951) alignment model by paramagnetic relaxation, Vrba et al. (1981) showed that the ratio $p / A_{\mathrm{V}}$ (equivalent to $p / I$ at millimetre wavelengths) should scale as $B^{2} a^{-1} n^{-1}$, where $B$ is the intensity of the magnetic field, $a$ the grain size, and $n$ the ambient density. If the magnetic field strength scales as $n^{1 / 2}$ in molecular clouds, as empirically observed (Crutcher 1999), the effect of the increased density and magnetic field cancel, and one is left with the dependence of $p / A_{\mathrm{V}}$ on the grain's size (and shape).

Fiege \& Pudritz (2000) showed that a helical magnetic field geometry in a cylindrical cloud with uniform grain properties could naturally produce a depolarization effect, in agreement with the submm polarization observations of the OMC-3 region of the Orion A filamentary molecular cloud (Matthews \& Wilson 2000; Matthews et al. 2001). Padoan et al. (2001) were able to produce a decreasing $p$ vs. $I$ relation in three-dimensional MHD simulations of supersonic and superAlfvénic turbulence assuming that grains are not aligned above
$A_{\mathrm{V}} \simeq 3 \mathrm{mag}$, but the dynamic range of the simulation did not extend much beyond this value of extinction. They noticed, however, that even assuming a uniform grain alignment efficiency, a decrease of $p$ with $I$ could be reproduced for particular orientations of the core magnetic field relative to the line of sight, an effect that will be further explored in the present paper.

It is possible in principle that the observed depolarization may be accounted for (at least in part) by beam smearing over tangled, small-scale field structures: Rao et al. (1998), for example, found that the decrease in polarization toward the Kleinman-Low nebula previously seen with single-dish observations was a result of subresolution-scale variations in the magnetic field that are averaged out by larger beams. However, given the relatively high levels of polarization detected at the core peaks, this effect should not be dominant.

We will try in this paper to further analyze the influence of the magnetic field geometry on the observed depolarization effect, studying cloud models dominated by a large scale magnetic field. In particular, we show that the pinching of the magnetic field expected in dense, self-gravitating molecular cloud cores naturally produces a decrease of the polarization degree toward the centre of the core, for a large range of viewing angles. In addition, we find that this geometrical depolarization effect is further enhanced by a dust temperature gradient increasing outward, as expected in externally heated starless cores (Evans et al. 2001; Zucconi et al. 2001; Stamatellos \& Whitworth 2003; Gonçalves et al. 2004, hereafter GGW). However, while these effects can contribute substantially to the observed depolarization, the measured $p-I$ relations are sometimes steeper than our predictions. Thus, we do not suggest that the field geometry and the dust temperature distribution are the sole means by which these observations could be explained: variations in the grain optical and/or geometrical polarization properties in the densest parts of a cloud are still required to account for the full range of observed polarization values.

The structure of the paper is the following: in Sect. 2, we describe the model adopted for magnetized molecular cloud cores and define the relevant physical and geometrical parameters of the problem; in Sect. 3, we show synthetic polarization maps computed with these models, and illustrate the effect of geometrical depolarization as well as its dependence on inclination, wavelength, and temperature distribution. In Sect. 4 we summarize our conclusions.

\section{The model}

We model molecular cloud cores as singular isothermal toroids, i.e. scale-free, axisymmetric equilibrium configurations of an isothermal gas cloud under the influence of self-gravity, gas pressure and magnetic forces ( $\mathrm{Li} \&$ Shu 1996). These toroids are characterized by one non-dimensional parameter, $H_{0}$, representing the fractional amount of support provided by magnetic forces. We show our polarization results for three models, with $H_{0}=0.2, H_{0}=0.5$ and $H_{0}=1.25$, corresponding to mass-to-flux ratios (in units of the critical value) $\lambda \approx 6,3$, and 1.6. With increasing $H_{0}$, the density distribution becomes flatter, and the configuration becomes a thin disk for $H_{0} \rightarrow \infty$. 


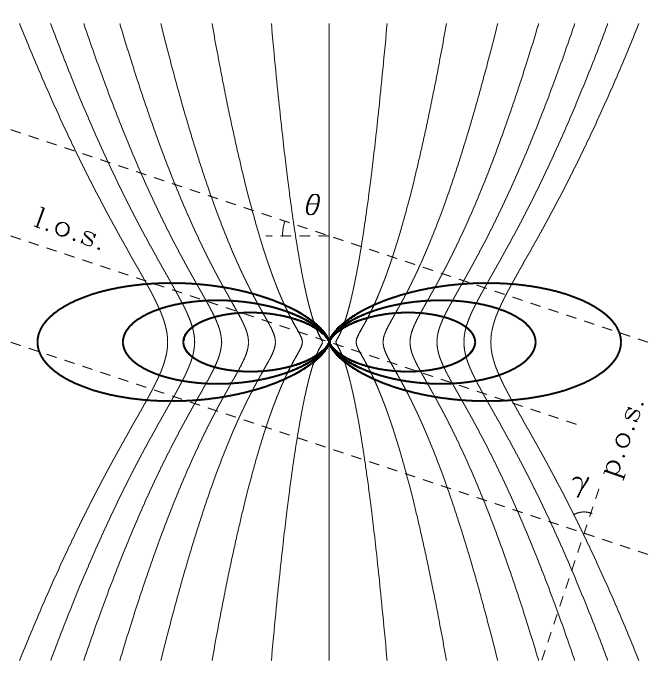

Fig. 2. The geometry of the model. The $H_{0}=1.25$ singular isothermal toroid (thick curves: isodensity contours; thin curves: magnetic field lines) is observed from a line of sight (l.o.s.) inclined by an angle $\theta$ with respect to the equatorial plane of the toroid $(\theta=0$ : edge-on; $\theta=90^{\circ}$ : pole-on). Dust grains are assumed to be aligned with the local direction of the magnetic field, making an angle $\gamma$ with the plane of the sky (p.o.s.). Notice that for the inclination shown in this figure, magnetic field lines are almost in the plane of the sky for lines of sight intercepting the toroid above and below the centre, resulting in a relatively higher polarization degree of the outer parts of the core with respect to the central region.

Figure 2 shows isodensity contours and magnetic field lines for a singular isothermal toroid with $H_{0}=1.25$, and illustrates some geometrical quantities adopted in our analysis.

We further assume that the core is bathed in the average interstellar radiation field (ISRF) of the solar neighborhood (Black 1994), and use the results of GGW for the temperature distribution. The dust temperature profile and the intensity of the emitted radiation at submillimetre wavelengths for the $H_{0}=0.5$ toroid were presented in Sect. 5 and Fig. 3 of GGW. We note some inconsistencies in our approach: first, the core's density distribution was obtained by Li \& Shu (1996) under the hypothesis that the gas is isothermal, whereas we explicitly consider deviations from isothermality at least in the dust component; second, GGW computed the dust temperature distribution assuming spherical grains, while in the present work, for the purpose of computing the polarization of the emitted radiation, we assume that the grains are aspherical and aligned with the magnetic field. However, the results of GGW are not substantially affected by grain shape.

Since we are interested in computing the sub-millimetre thermal emission, we neglect self-absorption of radiation. We also assume that the gas-to-dust ratio and the properties of the dust grains with respect to absorption and polarizing efficiency are uniform throughout the cloud. The polarization degree $p$ and polarization angle $\chi$ (the direction of polarization in the plane of the sky) are defined in terms of the standard Stokes parameters $Q, U$ and $I$,

$p=\frac{\sqrt{Q^{2}+U^{2}}}{I}$
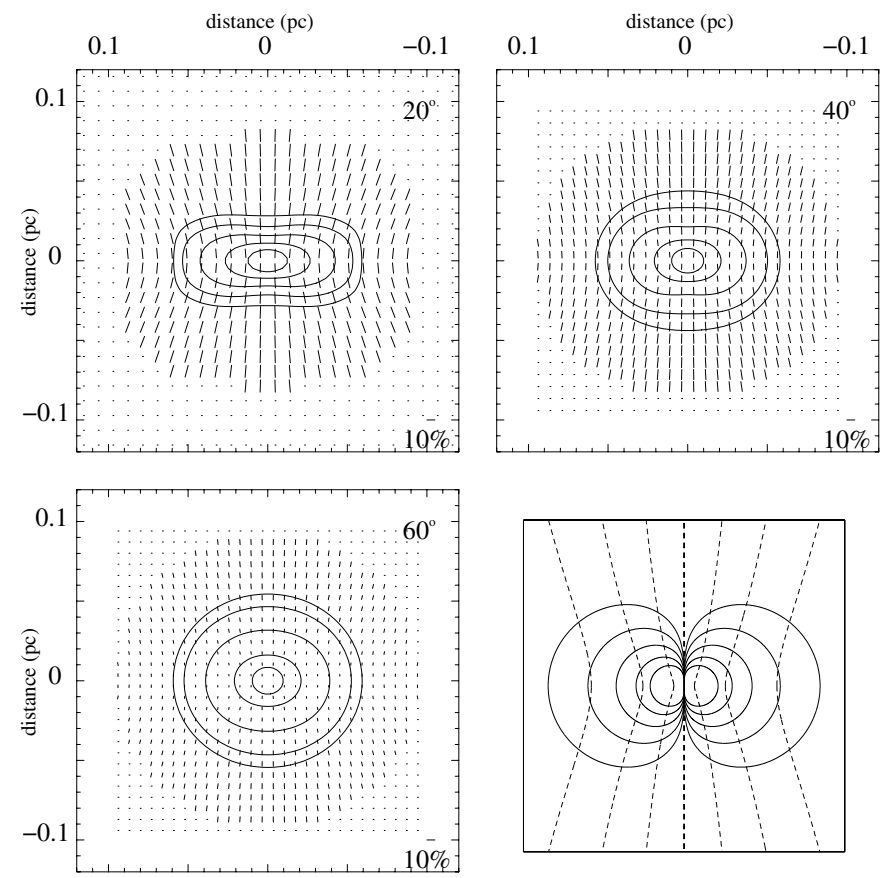

Fig. 3. Maps of the dust emission and polarization at $\lambda=850 \mu \mathrm{m}$ for the $H_{0}=0.2$ singular isothermal toroid, shown in the bottom right panel (solid curves, isodensity contours, dashed curves, magnetic field lines). The first three panels are for inclination with respect to the plane of the sky $\theta=20^{\circ}$ (top left panel), $\theta=40^{\circ}$ (top right panel), and $\theta=60^{\circ}$ (bottom left panel). Each vector is proportional to the polarization degree (see scale on the lower right corner of each panel), and has been rotated by $90^{\circ}$ to show the average orientation of the magnetic field in the plane of the sky. The intensity is shown by contours logarithmically spaced by 0.2 dex starting from $10 \%$ of the peak value.

$\tan 2 \chi=\frac{U}{Q}$

Here, we compute $p$ and $\chi$ following a method developed by Lee \& Draine (1985), and elaborated by Wardle \& Königl (1990), Fiege \& Pudritz (2000), and Padoan et al. (2001), but we generalize their method allowing a dependence of the dust temperature upon position inside the core. In this formulation, $p$ and $\chi$ are given by the simple expressions

$p=\alpha \frac{\sqrt{q^{2}+u^{2}}}{\Sigma-\alpha \Sigma_{2}}$,

$\tan 2 \chi=\frac{u}{q}$

where $\alpha$ is a nondimensional parameter representing the "polarizing efficiency" of dust grains, $q$ and $u$ are the "reduced" Stokes parameters

$q=\int \rho B_{\lambda}\left(T_{\mathrm{d}}\right) \cos 2 \psi \cos ^{2} \gamma \mathrm{d} \ell$

$u=\int \rho B_{\lambda}\left(T_{\mathrm{d}}\right) \sin 2 \psi \cos ^{2} \gamma \mathrm{d} \ell$ 
and

$\Sigma=\int \rho B_{\lambda}\left(T_{\mathrm{d}}\right) \mathrm{d} \ell$

$\Sigma_{2}=\int \rho B_{\lambda}\left(T_{\mathrm{d}}\right)\left(\frac{\cos ^{2} \gamma}{2}-\frac{1}{3}\right) \mathrm{d} \ell$.

These four quantities are integrals along the line of sight $\ell$ of the product of the total density $\rho$ (proportional, under our hypothesis, to the number density of dust grains), the Planck function $B_{\lambda}\left(T_{\mathrm{d}}\right)$ (representing the dust emissivity at the dust temperature $T_{\mathrm{d}}$ ), and a geometric factor accounting for the orientation of the magnetic field at each point inside the cloud, characterized by the two angles $\psi$ and $\gamma$. Specifically, the former is the angle between a direction in the plane of the sky (e.g. north) and the component of $\boldsymbol{B}$ in that plane; the latter is the angle between the local direction of $\boldsymbol{B}$ and the plane of the sky (see Fig. 2).

Thus, knowledge of the density and temperature distributions $\rho(\ell)$ and $T_{\mathrm{d}}(\ell)$, together with the geometry of the magnetic field in a cloud core expressed by $\psi(\ell)$ and $\gamma(\ell)$, are sufficient to completely determine the polarization characteristics of the radiation emitted by dust grains, if one specifies the nondimensional parameter $\alpha$ (constant under our assumptions), containing all information about the absorptions cross sections and the alignment efficiency. The numerical value of $\alpha$ can be easily fixed observing that the maximum polarization degree is achieved when $\boldsymbol{B}$ is in the plane of sky. In this case, with $\psi$ constant, Eq. (3) gives (Fiege \& Pudritz 2000)

$p_{\max }=\frac{6 \alpha}{6-\alpha}$,

or

$\alpha=\frac{6 p_{\max }}{6+p_{\max }} \approx p_{\max }$ if $p_{\max } \ll 1$.

The choice of $\alpha$ is therefore equivalent to a normalization of the expected degree of polarization. The polarization degree and angle can then be computed fixing the maximum polarization degree, instead of assuming specific grain properties. In this work we choose $\alpha=0.15$, which corresponds, from Eq. (9), to $p_{\max } \approx 15 \%$.

\section{Results}

For a molecular cloud core modeled as described in Sect. 2, with specified density and temperature distributions, the only free parameters are $\alpha$ and the inclination angle $\theta$ of the line of sight with respect to the equatorial plane of the toroid ( $\theta=0$ : edge-on; $\theta=90^{\circ}$ : pole-on). In this section we show the polarization of the emitted dust radiation for $\alpha=0.15$, varying the inclination of the toroid $\left(\theta=20^{\circ}, 40^{\circ}\right.$, and $\left.60^{\circ}\right)$, and for toroids with $H_{0}=0.2,0.5$ and 1.25 .

\subsection{Geometric depolarization at intermediate inclination}

In Fig. 3, we show the isophotes and polarization vectors (rotated by $90^{\circ}$ ) of the dust emission computed for the
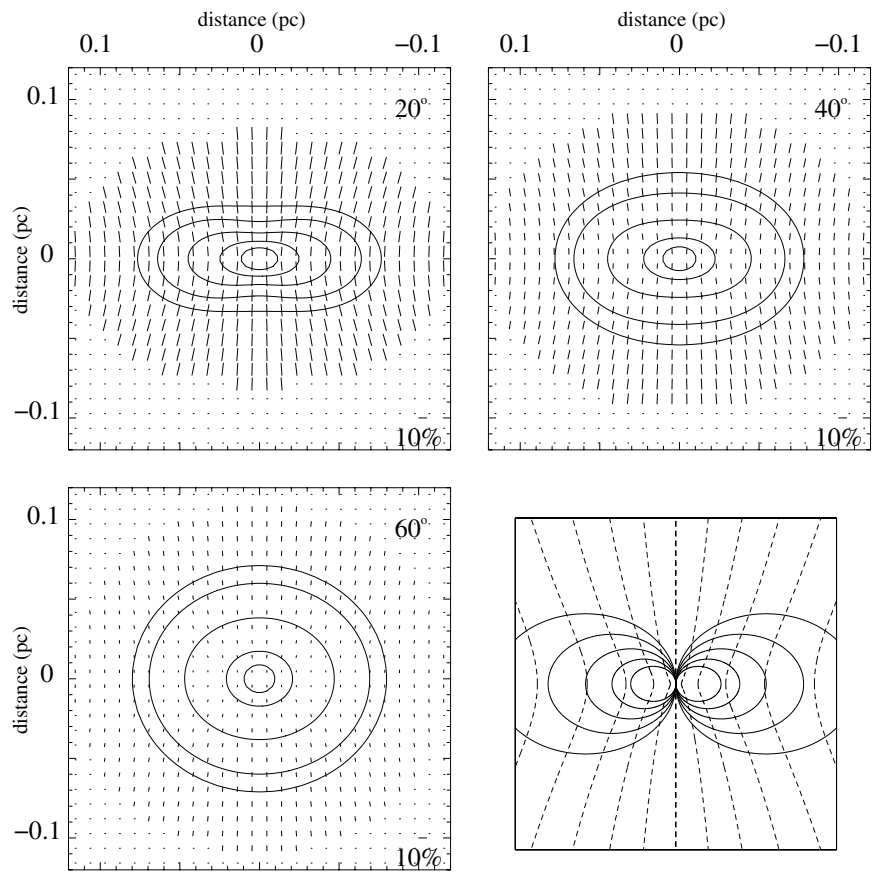

Fig. 4. Same as Fig. 3 for the $H_{0}=0.5$ singular isothermal toroid.
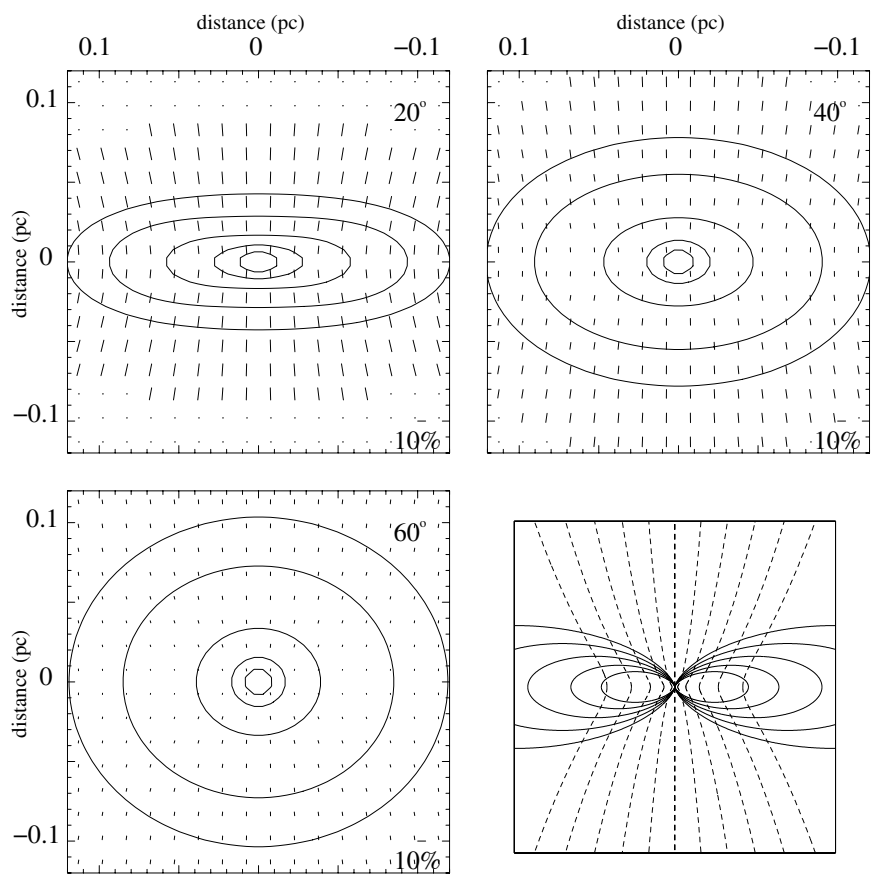

Fig. 5. Same as Figs. 3 and 4 for the $H_{0}=1.25$ singular isothermal toroid.

$H_{0}=0.2$ toroid at $\lambda=850 \mu \mathrm{m}$ for $\theta=20^{\circ}, 40^{\circ}$ and $60^{\circ}$. The model results have been convolved with a telescope beam of $F W H S$ of $12^{\prime \prime}$, assuming a distance of $150 \mathrm{pc}$ in all cases. Figures 4 and 5 show the same maps for the $H_{0}=0.5$ and 1.25 models, respectively. The polarization vectors have been rotated by $90^{\circ}$ to show the approximate average orientation of the magnetic field in the plane of the sky.

The polarization pattern is clearly symmetric, because of the assumed axial symmetry of the model. The equatorial pinching of the magnetic field lines (see Fig. 2) has an 


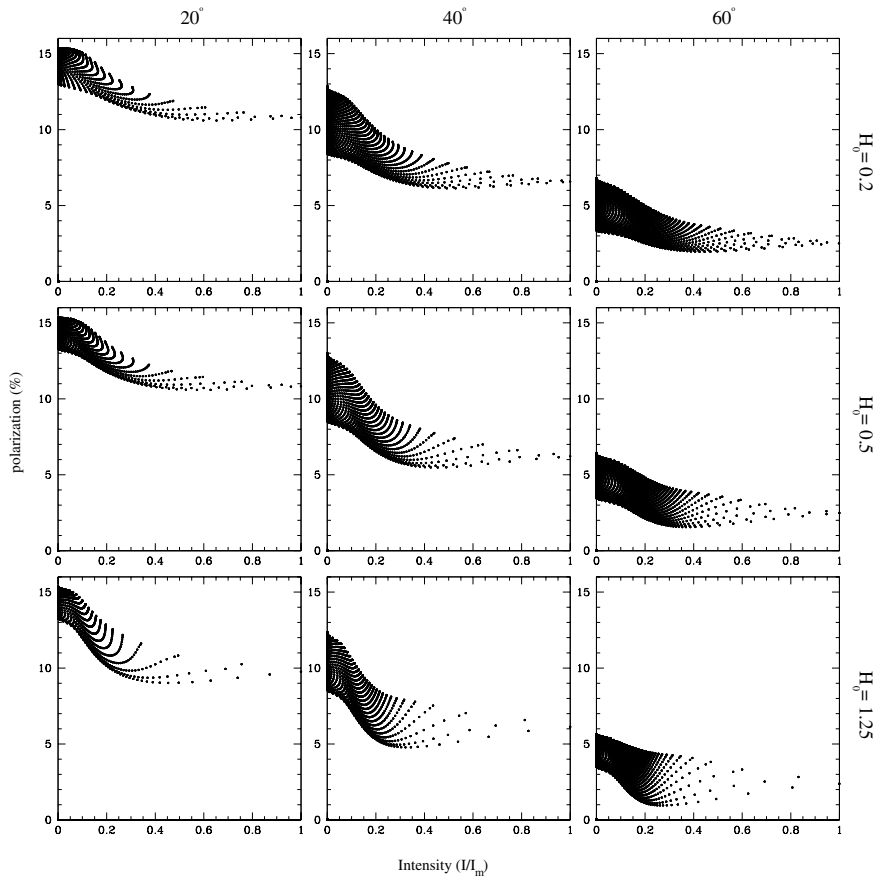

Fig. 6. Polarization degree as function of intensity at $850 \mu \mathrm{m}$ (normalized to the peak value $I_{\max }$ ) for the $H_{0}=0.2,0.5$ and 1.25 toroids for inclinations $\theta=20^{\circ}, 40^{\circ}$ and $60^{\circ}$. The multiple tracks visible in each panel are an artifact due to the sampling of the polarization degree over a regular square grid in the polarization maps.

important effect on the non-uniformity of the polarization degree across the cloud, resulting in a significant decrease of $p$ toward the central regions. In fact, for a hourglass magnetic field configuration observed at intermediate inclinations $(\theta \approx$ $30^{\circ}-40^{\circ}$ ), the largest component of the field in the plane of the sky is found in the outer parts of the cloud, whereas lines of sight close to the cloud's centre intercept regions where the bending of field lines is stronger and therefore the component of the field in the plane of the sky is relatively weaker (cf. Fig. 2).

The depolarization effect is better illustrated in Fig. 6, showing the degree of polarization $p$ as function of the intensity at $850 \mu \mathrm{m}$ (normalized to the peak intensity) for the $H_{0}=0.2,0.5$ and 1.25 toroids at three inclinations, $\theta=20^{\circ}$, $40^{\circ}$ and $60^{\circ}$. The minimum level of polarization is usually attained toward the centre of the core, where the non uniformity of the magnetic field along the line of sight is larger, and therefore cancellation effects more important. Over about one order of magnitude increase in intensity, the polarization degree decreases with roughly a power-law behavior. It is interesting to notice that even for a moderate pinching of the field (e.g. for the $H_{0}=0.2$ toroid), the decrease of polarization towards the centre is already significant.

We do not attempt in this paper to model specific objects, but we stress the qualitative similarity between our theoretical $p-I$ diagrams shown in Fig. 6 and the $p-I$ relations observed in dense cores (Fig. 1). In some cases, however, like in the dark cloud L183 (Crutcher et al. 2004) or L1544 (Ward-Thomson et al. 2000), the observed $p-I$ relation is too steep to be explained only on the basis of the field morphology and

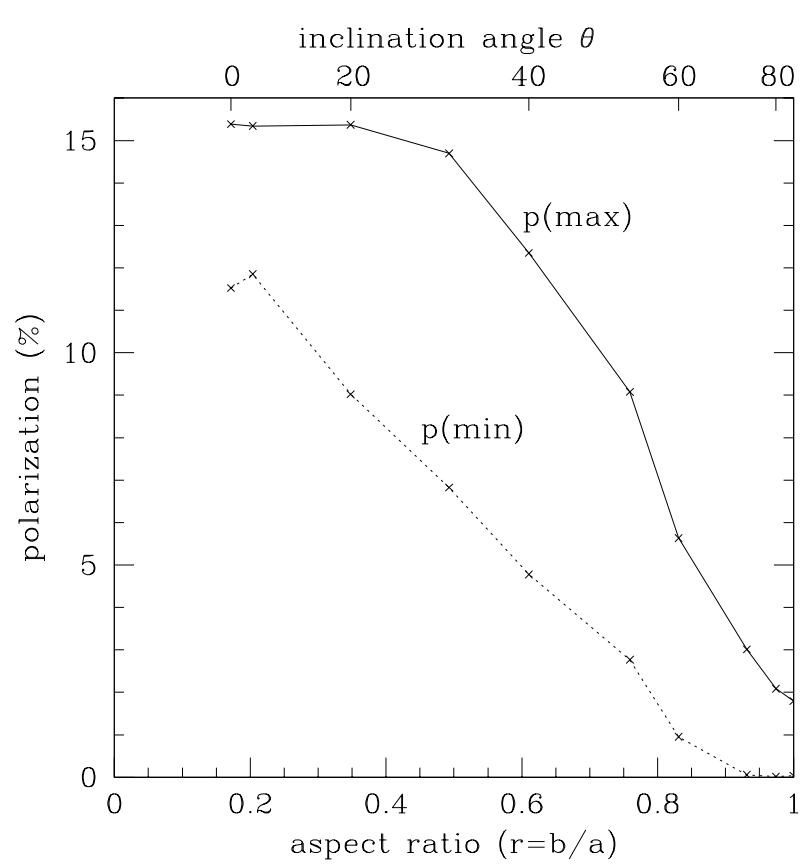

Fig. 7. Variation of maximum (solid line) and minimum (dotted line) polarization degree as function of the isophotal aspect ratio $r=b / a$ at $\lambda=850 \mu \mathrm{m}$ for the $H_{0}=1.25$ toroid. The upper scale shows the inclination angle $\theta$ (in degrees) of the line of sight with respect to the equatorial plane of the toroid.

inclination. A full model for the polarization of the emitted radiation probably requires additional mechanisms (such as an increase in size and sphericity of dust grains near the core centre) to reduce the polarizing efficiency $\alpha$ at high values of density or extinction.

\subsection{Dependence of depolarization on aspect ratio}

The maximum and minimum value of the polarization degree, $p_{\max }$ and $p_{\min }$, respectively, depend on the inclination angle $\theta$ of the core with respect to the line of sight. Both $p_{\max }$ and $p_{\min }$ decrease when the inclination of the toroid changes from edge-on $(\theta=0)$ to pole-on $\left(\theta=90^{\circ}\right)$, where $p_{\min }$ reaches zero. Correspondingly, the isophotal aspect ratio $r=b / a$ increases from a minimum value, that depends on the adopted model, to unity. For the three models considered in this paper, the minimum value of the aspect ratio is $r_{\min } \approx 0.6$ for the $H_{0}=0.2$ toroid, $r_{\min } \approx 0.4$ for the $H_{0}=0.5$ toroid, and $r_{\min } \approx 0.2$ for the $H_{0}=1.25$ toroid.

In Fig. 7 we show the maximum and minimum values of polarization as function of the aspect ratio $r$ for the $H_{0}=1.25$ toroid at $\lambda=850 \mu \mathrm{m}$. The inverse correlation shown by the figure between polarization degree and core aspect ratio is a characteristic signature of magnetic field configurations dominated by a poloidal component, and can be compared to observations to test the relative importance of poloidal vs. toroidal magnetic field components in dense clouds. In this sense, it is interesting to notice that of the three cloud cores represented in Fig. 1, the one showing the smallest range of variation (and absolute values) of $p$ (B1-c), is also the one characterized by the largest aspect ratio $(r \approx 1$, compared to $r \approx 0.5$ for $\mathrm{L} 183$ 


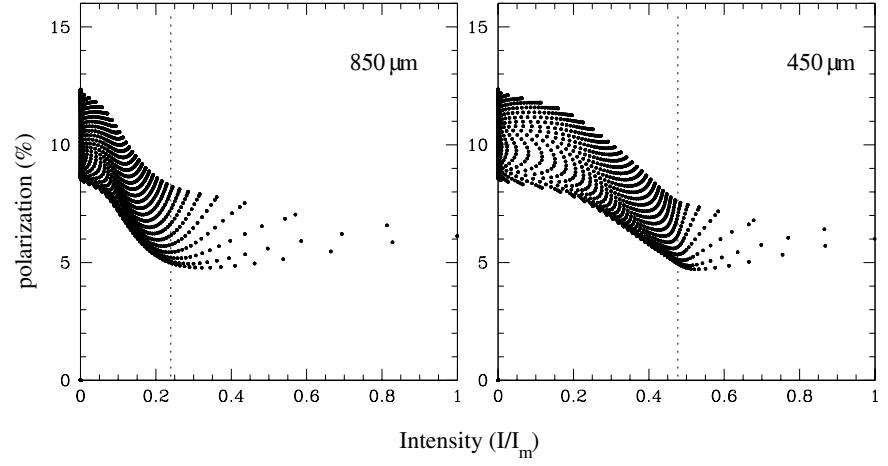

Fig. 8. Polarization degree as function of intensity at $850 \mu \mathrm{m}$ (left panel) and $450 \mu \mathrm{m}$ (right panel), normalized to the peak value $I_{\max }$, for the $H_{0}=1.25$ toroid at $\theta=40^{\circ}$. Notice the different extent of the "polarization hole", here identified with the region with low and uniform values of $p$ on the right side of the dashed lines: within $\sim 80 \%$ and $\sim 50 \%$ of the peak intensity at 850 and $450 \mu \mathrm{m}$, respectively.)

and L1544). A statistical analysis of the available observations may reveal the presence of a correlation between the range of polarization degree and the aspect ratio of cloud cores. As also shown by Fig. 7, the maximum depolarization effect, measured by $p_{\max }-p_{\min }$, is obtained when $r \approx 0.5\left(\theta \approx 30-40^{\circ}\right)$, and corresponds to a polarization reduction of a factor $\sim 2$, from $p_{\max } \approx 15 \%$ to $p_{\min } \approx 7 \%$. We notice that a detectable decrease in the polarization degree, say $p_{\max }-p_{\min }>5 \%$, occurs for inclinations ranging from $\sim 20^{\circ}$ to $\sim 65^{\circ}$. For random orientations of the toroids, this interval corresponds to about $50 \%$ of all possible cases.

\subsection{Dependence of depolarization on wavelength}

In Fig. 8, we compare polarization-intensity diagrams at $\lambda=$ $850 \mu \mathrm{m}$ and $\lambda=450 \mu \mathrm{m}$, obtained for the $H_{0}=1.25$ toroid at $\theta=40^{\circ}$. As shown by the figure, the minimum and maximum value of polarization remain the same at these two wavelengths, but their dependence on intensity is different, the decrease of $p$ with $I$ being steeper at the longer wavelength. This effect can be easily understood: since the dust emission at $\lambda=450 \mu \mathrm{m}$ is less concentrated than at $\lambda=850 \mu \mathrm{m}$ (see e.g. GGW), a given value of $I / I_{\max }$ corresponds to a larger distance from the centre of the core (the intensity peak) at the shorter wavelength. Therefore the "polarization hole" appears restricted to higher values of $I / I_{\max }$ at $\lambda=450 \mu \mathrm{m}$.

\subsection{Effects of a non isothermal dust temperature distribution}

Figure 9 shows the importance of computing the dust temperature distribution resulting from the heating of the external ISRF instead of assuming an uniform dust temperature through the cloud. In fact, the outwardly increase of the dust temperature from $\sim 8 \mathrm{~K}$ to $\sim 15 \mathrm{~K}$ for an externally heated cloud (see GGW) enhances, in the integration along the line of sight, the contribution to the Stokes parameters of the external layers of the cloud, where, as we have seen, the component of the magnetic field in the plane of the sky is generally larger than near the core's

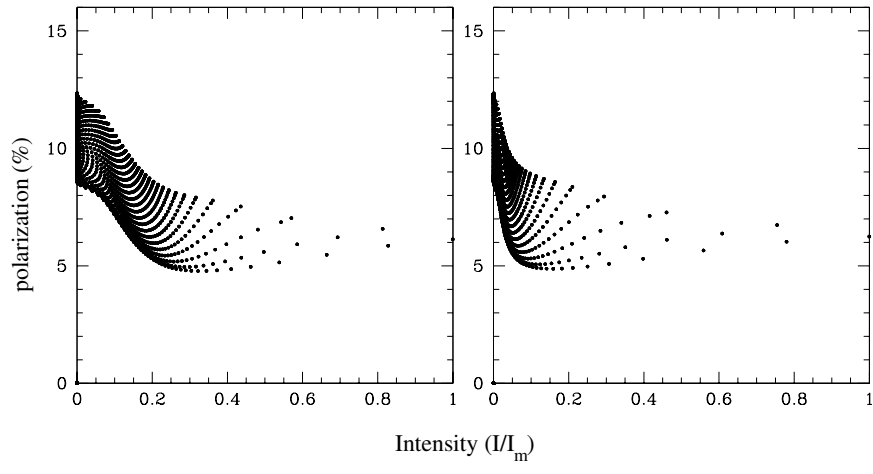

Fig. 9. Polarization degree as function of intensity at $850 \mu \mathrm{m}$ (normalized to the peak value $I_{\max }$ ) for the $H_{0}=1.25$ toroid at $\theta=40^{\circ}$, in the case of external heating by the ISRF (left panel) and assuming an isothermal dust temperature distribution (right panel).

centre. Thus, an outward increasing temperature gradient contributes to the observability of the depolarization effect. In fact, given the current sensitivity of polarimeters at submillimetre wavelengths, that allow measurements of $p$ only for $I \gtrsim 0.2 I_{\max }$ (see Fig. 1), the value of $p$ would be quite uniform through the sampled region if the cloud were isothermal (see Fig. 9). Thus, the decrease of polarization shown by our model is actually the result of the combination of pinched magnetic field hourglass and a dust temperature gradient increasing outward.

\subsection{Effects of turbulence}

Finally, we remark that the predicted $p-I$ diagrams of condensations formed in simulations of turbulent flows (Padoan et al. 2001) differ significantly from our Fig. 6, as they show a large population of low- $p$ and low- $I$ data points, which, in general, is not observed (cf. Fig. 1). Thus, at least in principle, $p-I$ diagrams offer a way to distinguish between different explanations of the observed behavior of polarization in cloud cores, especially given the increased sensitivity expected from the next generation of detectors and polarimeters.

In addition, our results show that a large-scale magnetic field with moderate equatorial pinching can produce a significant deviation of polarization angles from the direction of the cloud's minor axis, up to about $\pm 15^{\circ}$ (see Figs. $3-5$ ). For example, in the case of the $H_{0}=0.2$ toroid observed at an inclination $\theta=20^{\circ}$ (Fig. 2), the distribution of polarization angles over the whole map is peaked on the direction of the cloud's minor axis (a consequence of the assumed axial symmetry of the model), but the dispersion in polarization angles around the mean direction is $\sigma_{\chi} \approx 10^{\circ}$. This casts doubt on the use of the Chandrasekhar-Fermi formula to estimate the magnetic field strength in molecular cloud cores, as this formula assumes that observed deviations of polarization angles from a given direction (of the order of $\sigma_{\chi} \approx 10^{\circ}-15^{\circ}$ in starless cores, see e.g. Crutcher et al. 2004) are solely due to the presence of a turbulent (or better, "wavy") component of the field. 


\section{Conclusions}

In this paper we have computed the polarization of the radiation emitted by dust grains in molecular cloud cores represented as magnetically supported equilibrium configurations. To this end, we have adopted the magnetostatic models of Li \& Shu (1996) and the radiative transfer method developed by GGW. The Stokes parameters (and therefore the polarization degree) have been computed at two wavelengths $(\lambda=$ $850 \mu \mathrm{m}$ and $\lambda=450 \mu \mathrm{m}$ ) and for various inclinations of the cloud with respect to the line of sight, assuming that the dust grains are elongated and aligned with the large-scale magnetic field.

The main result of this paper is the demonstration that a significant depolarization effect, with characteristics very similar to those observed in actual cloud cores, can arise only because of geometrical effects, if the large-scale magnetic field has the equatorially pinched morphology predicted by magnetically dominated models. However, we do not claim that the field geometry is the sole means by which such an effect could be produced. There may also be contributions due to grain growth in the densest parts of a cloud, and turbulence. We also note that the assumption of axial symmetry of the density and field distribution implies that the model polarization vectors (after a $90^{\circ}$ rotation), are symmetrically distributed with respect to the cloud's minor axis, at variance with observational evidence for some cores (see e.g. Ward-Thompson et al. 2000). A toroidal component of the magnetic field, ignored in the present analysis, can in principle account for the observed misalignment between the core apparent elongation and the average polarization position angle (see e.g. Vallée et al. 2003).

Acknowledgements. We thank Richard Crutcher for interesting discussions and for providing polarization data for starless cores. JG acknowledges support from the scholarship SFRH/BD/6108/2001 awarded by the Fundação para a Ciência e Tecnologia (Portugal).

\section{References}

Crutcher, R. M., Nutter, D. J., Ward-Thompson, D., \& Kirk, J. M. 2004, ApJ, 600, 279

Davis, L. Jr., \& Greenstein, J. L. 1951, ApJ, 114, 206

Draine, B. T., \& Weingartner, J. C. 1996, ApJ, 470, 551

Draine, B. T., \& Weingartner, J. C. 1997, ApJ, 480, 633

Evans, N. J., Rawlings, J. M. C., Shirley, Y., \& Mundy, L. G. 2001, ApJ, 557, 193

Fiege, J. D., \& Pudritz, R. E. 2000, ApJ, 544, 830

Gonçalves, J., Galli, D., \& Walmsley, C. M. 2004, A\&A, 415, 617 (GGW)

Henning, Th., Wolf, S., Launhardt, R., \& Waters, R. 2001, ApJ, 561, 871

Hildebrand, R. H., Dotson, J. L., Dowell, C. D., Schleuning, D. A., \& Vaillancourt, J. E. 1999, ApJ, 516, 834

Lai, S.-P., Girart, J. M., \& Crutcher, R. M. 2003, ApJ, 598, 392

Lazarian, A., Goodman, A. A., \& Myers, P. C. 1997, ApJ, 490, 273

Lee, H. M., \& Draine, B. T. 1985, ApJ, 290, 211

Li, Z.-Y., \& Shu, F. H. 1996, ApJ, 472, 211

Matthews, B. C., \& Wilson, C. D. 2000, ApJ, 531, 868

Matthews, B. C., \& Wilson, C. D. 2002, ApJ, 574, 822

Matthews, B. C., Wilson, C. D., \& Fiege, J. D. 2001, ApJ, 562, 400

Padoan, P., Goodman, A., Draine, B. T., et al. 2001, ApJ, 559, 1005

Rao, R., Crutcher, R. M., Plambeck, R. L., \& Wright, M. C. H. 1998, ApJ, 502, 75L

Stamatellos, D., \& Whitworth, A. P. 2003, A\&A

Vallée, J. P., Greaves, J., \& Fiege, J. D. 2003, ApJ, 588, 910

Vrba, F. J., Tapia, S., \& Coyne, G. V. 1981, ApJ, 243, 289

Vrba, F. J., Coyne, G. V., \& Tapia, S. 1993, AJ, 105, 1010

Wardle, M., \& Königl, A. 1990, ApJ, 362, 120

Ward-Thompson, D., Kirk, J. M., Crutcher, R. M., et al. 2000, ApJ, 537, L35

Wolf, S., Launhardt, R., \& Henning, T. 2003, ApJ, 592, 233

Zucconi, A., Walmsley, C. M., \& Galli, D. 2001, A\&A, 376, 650 